\title{
Application of Fuzzy Model Predictive Control in Multivariable Control of Distillation Column
}

\author{
R. Sivakumar, K. Suresh Manic, V. Nerthiga, R. Akila, K. Balu
}

\begin{abstract}
In this paper, a fuzzy model predictive control strategy is proposed for multivariable nonlinear control problem in a distillation column. This method is based on piecewise linear fuzzy model of the process to be controlled, which is used for predicting the outputs. An optimization technique is developed to minimize the difference between the model predictions and the desired prediction horizon. Comparisons are made with conventional controllers. The results confirmed the potentials of the proposed strategy of piecewise linear fuzzy control.
\end{abstract}

Index Terms - Distillation column, MIMO systems, Model Predictive Control, Takagi-Sugeno fuzzy model.

\section{INTRODUCTION}

Model Predictive Control (MPC) is a powerful tool for the control of multivariable systems. It has become a popular research topic during last few decades [1] and unlike many other advances techniques. The main reason for this success is the ability of MPC to control multivariable systems under various constraints in an optimal way.

Continuous and batch processes in chemical and petrochemical plants are inherently nonlinear and many of them are highly nonlinear. For highly nonlinear system, a linear MPC algorithm may not give satisfactory dynamic performance. Several researchers [10] have developed nonlinear model predictive control (NMPC) algorithms that accept various kinds of nonlinear models such as nonlinear ordinary differential equations, partial differential equations, integro-differential equations and delay equations models. Such models can be accurate over a wide range of operating conditions. However, these models, usually based on the first principles, are difficult to develop for many industrial cases. Moreover, an NMPC incorporating a nonlinear process can be precisely described by a set of linear submodels in someway, and then the design of a model predictive controller can be greatly simplified.

Reference [3] introduced a novel fuzzy logic-based modeling methodology, where a nonlinear system is divided into a number of linear or nearly linear subsystems. A

Manuscript received April 9, 2010.

R. Sivakumar is with St. Joseph's College of Engineering, Anna University, Chennai ( phone: +91 9444309944; fax: +91 442450 0861; e-mail: rsivakumar1@gmail.com).

K. Suresh Manic is with St. Joseph's College of Engineering, Anna University, Chennai (e-mail: ksuresh_me@yahoo.com)

V. Nerthika is a research scalar in the Department of Chemical engineering, Anna University, Chennai (e-mail: nerthi82@gmail.com)

R. Akila is a research scalar in the Department of Chemical engineering, Anna University, Chennai (e-mail: akilarajamanickam@yahoo.com)

$\mathrm{K}$. Balu is with Department of Chemical Engineering, Anna University, Chennai (e-mail: kbalu@annauniv.edu) quasi-linear empirical model is then developed by means of fuzzy logic for each subsystem. The model is a rule-based fuzzy implication (FI). The whole process behavior is characterized by a weighted sum of the outputs from all quasi-linear FIs. The methodology facilitates the development of a nonlinear model that is essentially a collection of a number of quasi-linear models regulated by fuzzy logic. It also provides an opportunity to simplify the design of model predictive controllers.

However some issues limit the possible application of MPC to multivariable systems with significant delays and nonlinear systems. These limitations can be deal with fuzzy MPC (FMPC). However, tremendous difficulties have been found in tuning controller parameters since the algorithm requires frequent model updating in control. A T-S type model is the basis of their fuzzy model. However, they essentially treated the fuzzy model as a set of conventional piecewise linear models. Thus, the uniqueness of a Takagi-Sugeno-type model exhibiting soft transition through any operating regions is lost, causing deterioration in the closed-loop dynamic performance of a system.

The recent interest in fuzzy logic controllers can be attributed to their ability to exploit the tolerance for imprecision and uncertainty to achieve robustness and low-cost solution. The advantages of fuzzy logic are used with MPC to provide the solution for complex problems. In this paper a FMPC algorithm is developed and applied to binary distillation column. First, a fuzzy model of distillation column is developed and can be used as a predictor in MPC [5]. Then the objective functions and optimizer based on fuzzy rules are developed.

Model based controllers use an internal model to predict future outputs. These future outputs can be calculated by means of different optimization methods, depending on the system and objective functions. In this paper, TakagiSugeno fuzzy model is used for distillation column and branch-and-bound algorithm is used for optimization.

\section{PROCESS DESCRIPTION}

\section{A. System Modelling}

A typical two product distillation column is taken as study model shown in Fig. 1 shows the most important loops of a binary distillation. Acceptable operation of a binary distillation column normally requires the following control objects:

- Control of the composition of the distillate

- Control of the composite of the bottom products

- Control of the liquid hold-up in reflux drum

- Control of the liquid hold-up at the base of the column

The first two control objectives characterize the two 
product streams, where the other two objects are required for operational feasibility [7] (i.e to prevent flooding and drying up of the reflux drum and the base of the column). The dynamic responses of control loop 3 and 4 in fig. 1 are usually much faster than the dynamic responses of other control loops

A mathematical model of a binary distillation column based on various simplifying assumptions was used in our studies. In the development of the mathematical model, the dynamics introduced by control loop 3 and 4 have been neglected and the hold up of liquid in the reflux drum and the base of the column have been assumed to be constant. It has also been assumed that the quality of the distillate is controlled by manipulating the reflux flow rate, whereas the bottom product quality is controlled by manipulating the boilup rate.

Control of boilup rate is normally exercised by varying the steam flow rate to the reboiler. In the mathematical model, the dynamics of heat transfer processes in the condenser and the reboiler are neglected. In commercial scale columns, the dynamic response of these heat exchangers is usually much faster than the response of the column itself. To control the entire loop with interaction loop many variables multi loop control scheme always used. This multivariable control strategy may be implemented for either time domain or Laplace transform models. For multivariable systems such as distillation columns having multiple delays, a commonly employed linear model takes the form:

$$
y(s)=G p(s) u(s)+G d(s) d(s)
$$

Where $\mathrm{y}$ is a vector of outputs, $\mathrm{u}$ a vector of controls and $\mathrm{d}$ a vector of disturbance variable.

The transfer function of distillation column used in our studies is

$$
\left[\begin{array}{l}
Y_{1} \\
Y_{2}
\end{array}\right]=\left[\begin{array}{cc}
\frac{12 e^{-s}}{16.7 s+1} & \frac{-18.9 e^{-2 s}}{12 s+1} \\
\frac{6.6 e^{-7 s}}{19.9 s+1} & \frac{-19.4 e^{-3 s}}{14.4 s+1}
\end{array}\right]\left[\begin{array}{l}
u_{1} \\
u_{2}
\end{array}\right]
$$

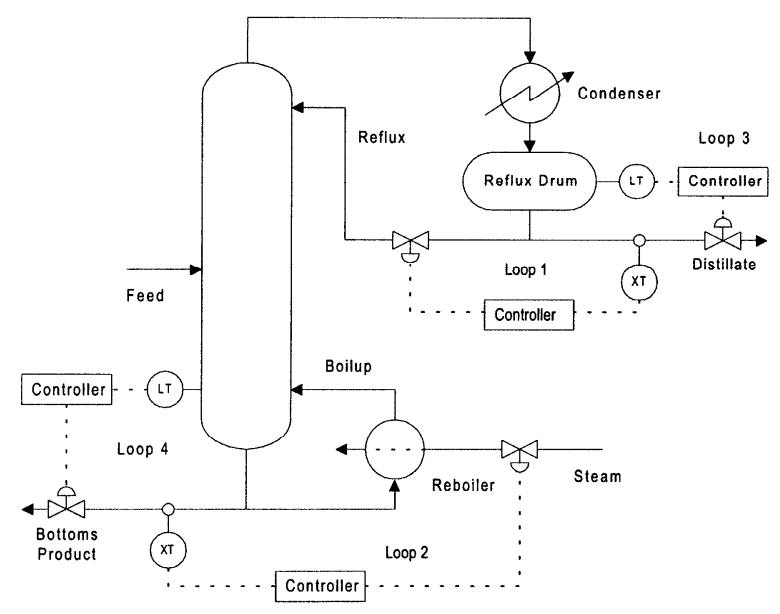

Fig. 1. Control of Binary Distillation Column

\section{B. Piecewise Linear Fuzzy Model}

A fuzzy model is the name of systems which use some concepts from fuzzy logic (fuzzy sets, linguistic variables, etc.). An important difference compared to other modelling techniques is that they can easily incorporate knowledge which is provided by human experts and they do not depend only on numerical data collected from the process. Engineering fuzzy models should have real valued input and output variables and are classified into two main categories: Mamdani models and Takagi-Sugeno models [2]. The main difference between the above two categories can be found in the consequent parts of the fuzzy rules. In the Mamdani models the THEN part is fuzzy, while in the Takagi-Sugeno case the consequent part is crisp and is expressed as a linear combination of the input variables. Takagi and Sugeno fuzzy models are suitable to model a complex system. Fuzzy modelling and identification from measured data are effective tools for the approximation of uncertain multivariable systems. In a dynamic fuzzy model, at time point $\mathrm{k}$, past values of the process input and output variables constitute the input variables to the system. We will use only input variables, provided that enough past values will be considered in the model. The fuzzy model is structured as follows.

$$
\begin{aligned}
& \text { Li: } \quad \begin{aligned}
& \text { IF } y(t) \text { is } B_{i} \text { THEN } \\
& y m(t+1)=-a_{i 1} y(t)-\cdots-a_{i j} y(t-j+1) \\
&+b_{i 1} u(t)+\cdots+b_{i 1} u(t-1+1)
\end{aligned}
\end{aligned}
$$

Where $y(t)$ is the process output, $u(t)$ is the process inputs, and $y m(t+1)$ is the one step ahead model prediction at time $t$ : $\mathrm{Bi}$ is a fuzzy set representing the fuzzy sub-space in which implication Li can be applied for reasoning; and $i=1, \ldots, p$. the model parameters can be represented by the matrix $\Phi$ as follows

$$
\Phi=\left|\begin{array}{ccccc}
a_{1}^{l} \ldots a_{j}^{l} & b^{l} \ldots b_{l}^{l} & k_{l} \\
\ddots & & & \\
a_{1}^{p} \ldots a_{j}^{p} & b^{p}{ }_{1} \ldots & b^{p}{ }_{l} & k_{p}
\end{array}\right|
$$

When a set of input-output data is given, the model parameters can be calculated using the method of least squares. The method as proposed by Takagi - Sugeno involves an iterative search to determine the best model structure, the optimum fuzzy partitioning and parameter estimation. The overall model fit is assessed using a performance index such as mean square prediction error based on the test data. It is possible to express the overall fuzzy model output in the following form:

$$
\mathrm{y}_{\mathrm{m}}(\mathrm{t}+1)=\beta \Phi \mathrm{X}(\mathrm{t})
$$

where

$$
\begin{gathered}
X(t)=\left[\begin{array}{c}
-y(t) \ldots-y(t-j+1) \\
u(t) \ldots u(t-1+1) \quad 1
\end{array}\right] T \\
\beta=\left[\begin{array}{llll}
\beta 1 & \ldots & \beta i & \ldots p
\end{array}\right]
\end{gathered}
$$


and

$$
\beta=\frac{B_{i}[y(t)]}{\sum_{i=1}^{p} B_{i}[y(t)]}
$$

$\operatorname{Bi}[y(t)]$ is the grade of membership of $y(t)$ in $\mathrm{Bi}$ and $\beta$ is $\mathrm{s}$ vector of the weights to each of the $p$ implications at each sampling instant.

\section{Classical MPC}

The main idea behind MPC-type controllers is illustrated in Fig. 2 for a SISO system. At sampling time $\mathrm{k}$, a set of $\mathrm{m}$ future manipulated variable moves (control horizon) are selected, so that the predicted response over a finite horizon $p$ (prediction horizon) has certain desirable characteristics. This is achieved by minimizing an objective function based on the deviation of the future controlled variables from a desired trajectory over the prediction horizon $\mathrm{p}$ and the control energy over the control horizon $\mathrm{m}$. The MPC optimization is performed for a sequence of hypothetical future control moves over the control horizon and only the first move is implemented [4].

The problem is solved again at time $\mathrm{k}+1$ with the measured output $y(k+1)$ as the new starting point. Model uncertainty and unmeasured process disturbances are handled by calculating an additive disturbance as the difference between the process measurement and the model prediction at the current time step. For the measured disturbances it is assumed that the future values will be equal to the current values. The performance of MPC depends largely on the used process model. The model must be able to accurately predict the future process outputs, and at the same time be computationally attractive to meet real-time demands.

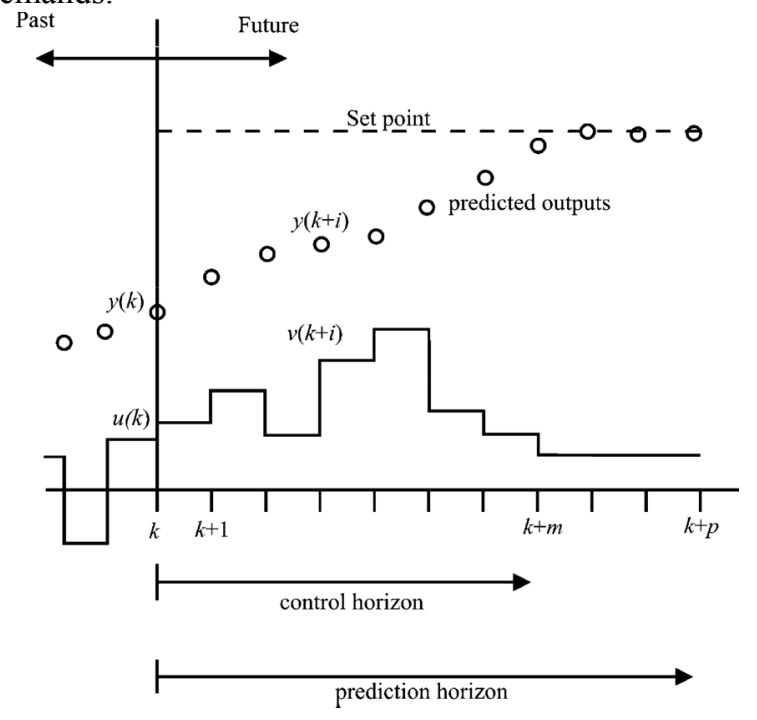

Fig. 2. A schematic view of MPC

\section{FMPC OPTIMIZATION}

Fig. 2 gives the general idea of a general model predictive control. The MPC optimization problem can be formulated as a search in the discrete space of control actions. A discrete optimization method is used to find an optimal control action. The branch-and-bound method is a structured search technique that belongs to a general class of combinatorial programming methods. The branch-and-bound method solves a problem by dividing it into sub-problems, using a tree structure [4]. This method is based on the fact that, in general, only a small number of the possible solutions actually need to be enumerated, so the remaining solutions are eliminated through the applications of bounds. i.e., the objective function is used to decide whether a branch is further examined or not.

Branch-and-bound has been applied successively to classical predictive control problems. this method the set of solutions is subsequently partitioned into increasingly refined parts (branching) over which lower and upper bounds for the optimal value of the objective function can be determined (bounding). The uses of this discrete technique do not avoid, however, the complexity of the optimization problem. Note that the branch-and-bound algorithm applied to MPC, can be extended by including fuzzy decision criteria in the objective function.

The branch-and-bound method can only be applied to predictive control when the control actions are discretized. The model of the system under control predict the future outputs of the system $y(k+1), \ldots, y(k+H p)$, and is given by

$$
y(k+i)=f(x(k+i-1), u(k+i-1), i=1, \ldots, H p .
$$

Let the possible inputs of the system be discretized in $\mathrm{M}$ possible control actions. Let also the discretized control actions be denoted $\omega_{\mathrm{j}}$. Thus, at each step the control actions $\mathrm{u}(\mathrm{k}+\mathrm{i}-1) \in \Omega$, are given by

$$
\Omega=\left\{\omega_{j} \mid j=1,2, \ldots M\right\}
$$

The problem to be solved in MPC is represented by an objective function. This optimization problem is successively decomposed by the branching rule into smaller sub-problems.

At time instant $\mathrm{k}+\mathrm{i}$ the cumulative cost of a certain path followed so far, and leading to the state $\mathrm{x}(\mathrm{k}+\mathrm{i})$ and output $\mathrm{y}(\mathrm{k}+1)$ is given by

$$
J(u)=\sum_{i=1}^{p}(r(k+i)-y(k+i))^{2}+\beta(\Delta u(k+i-1))^{2}
$$

where $\mathrm{i}=1, \ldots \mathrm{Hp}$ denotes the level corresponding to the time step $\mathrm{k}+\mathrm{i}$ A particular branch $\mathrm{j}$ at level $\mathrm{i}$ is created when the cumulative cost J(i) plus a lower bound on the cost from the level $\mathrm{i}$ to the terminal level Hp for the branch $\mathrm{j}$ denoted $\mathrm{JLj}$ is lower than an upper bound of the total cost, denoted JU :

$$
\mathrm{J}(\mathrm{i})+\mathrm{JLj}<\mathrm{JU}
$$

The branch-and-bound algorithm applied to MPC always finds the global discrete optimal solution, guaranteeing a good control performance when the number of control actions $\mathrm{M}$ is sufficiently large. Moreover, the branch-and-bound method implicitly deals with constraints. In fact, the presence of constraints improve the efficiency of 
bounding, restricting the search space by eliminating non-feasible sub-problems

\section{RESUlTS AND DisCUSSION}

Because the A Wood and Berry binary distillation column model is taken for our study. First we applied conventional PID controller which is tuned by BLT method and then FMPC. Fig. 3 shows the responses of binary distillation column using FMPC. The control performance of this process is compared with conventional PID controller tuned with BLT method is shown in fig. 4,5 and the results are tabulated in the table 1. Almost the performances by FMPC method are better than that conventional technique. Only one point the FMPC method of control has negative overshoot.

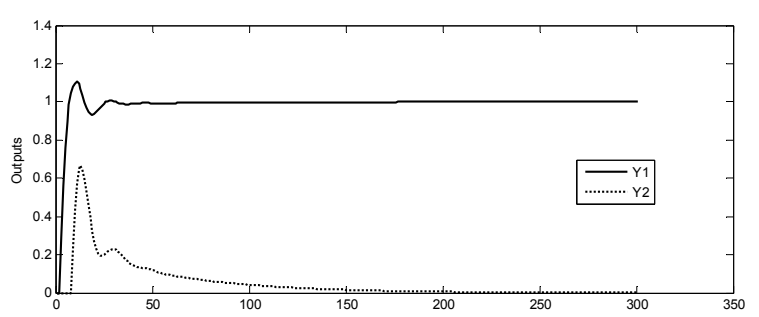

(a)

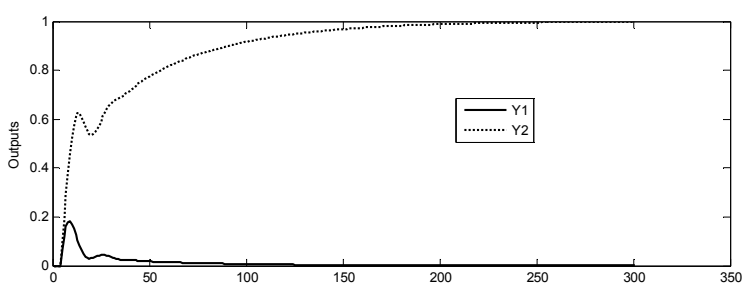

(b)

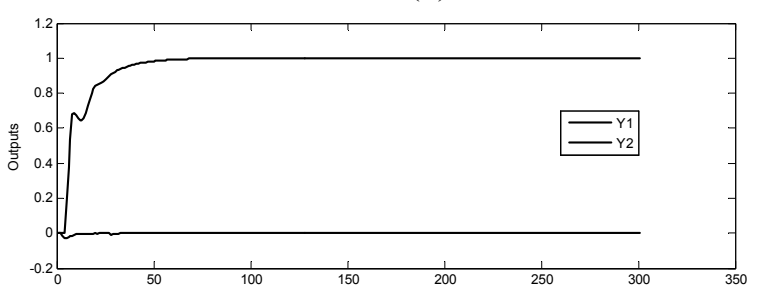

(c)

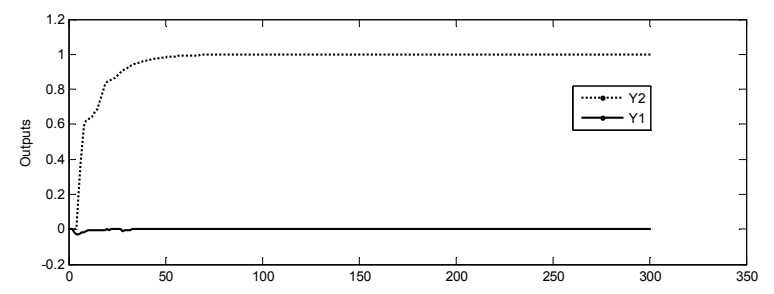

(d)

Fig. 3. Responses of distillation column: step change in $y_{1}(a)$, $\mathrm{y}_{2}(\mathrm{~b})$ using PID controller. Step change in $\mathrm{y}_{1}(\mathrm{c}), \mathrm{y}_{2}(\mathrm{~d})$ using FMPC

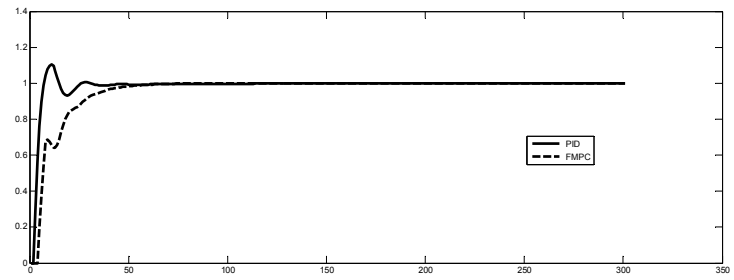

Fig. 4. Comparison of conventional PID controller and Fuzzy Model Predictive Controller for step change in $\mathrm{y}_{1}$.

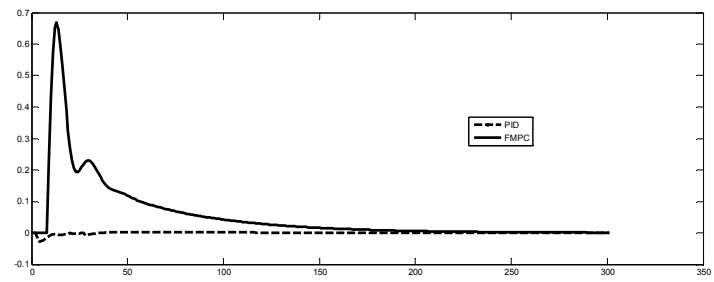

Fig. 5. Comparison of conventional PID controller and Fuzzy Model Predictive Controller for step change in $\mathrm{y}_{2}$

Table 1. Performance Comparison

\begin{tabular}{|l|l|l|l|l|l|}
\hline \multirow{2}{*}{$\begin{array}{l}\text { Step } \\
\text { change }\end{array}$} & \multirow{2}{*}{$\begin{array}{l}\text { Controlled } \\
\text { Variable }\end{array}$} & \multicolumn{3}{l}{$\begin{array}{l}\text { Settling time } \\
\text { in Sec }\end{array}$} & \multicolumn{2}{l|}{ Overshot } \\
\cline { 3 - 6 } & & PID & FMPC & PID & FMPC \\
\hline \multirow{2}{*}{ y1 } & $\mathrm{y} 1$ & 67 & 61 & 0.105 & 0.02 \\
\cline { 2 - 6 } & $\mathrm{y} 2$ & 172 & 43 & 0.67 & -0.05 \\
\hline \multirow{2}{*}{ y2 } & $\mathrm{y} 1$ & 163 & 45 & 0.182 & -0.03 \\
\cline { 2 - 6 } & $\mathrm{y} 2$ & 176 & 66 & 0.01 & 0.01 \\
\hline
\end{tabular}

\section{CONCLUSION}

Fuzzy model predictive controller offers better control responses than conventional PID controllers for multivariable processes. Application of FMPC of distillation column gives a fairly performance. Simulation results were carried out for different servo problems. The results indicate that improve for hybrid controllers.

\section{REFERENCES}

[1] G. R. Arulalan and P. B. Deshpande, "Simplified model predictive control,"Ind. Eng. Chem. Res., vol. 26, pp. 356-362, 1987.

[2] J.A. Roubos et. al (1999), "Fuzzy model-based predictive control using Takagi - Sugeno models", International Journal of Approximate Reasoning, Vol. 22, pp. 3 - 30, 1999.

[3] T. Takagi and M. Sugeno, "Fuzzy identification of systems and its application to modeling and control," IEEE Trans. Syst., Man, Cybern., vol. SMC-15, pp. 116-132, Jan./Feb. 1985.

[4] Piotr Tatjewski and Maciej Lawryn' Czuk (2006), "Soft computing in model-based predictive control", International Journal of Applied Maths and Computer Science, Vol. 16, No. 1, pp. 7-26, 2006

[5] Fabrizio Bezzo et. al (2005), "Using MPC to control middle-vessel continuous distillation column", Journal of Process Control, Vol. 15, pp. 925-930, 2005

[6] M. Mahfouf et. al (2002), 'Fuzzy model-based predictive control using an ARX structure with feedforward', Fuzzy Sets and Systems, Vol. 125, pp.39-59, 2002

[7] S. Skogestad,(1997), ' Dynamics and control of distillation columns: A tutorial introduction', Transaction of Institution of Chemical Engineers, Vol. 75, part A, pp. 539-562, 1997.

[8] A. Rueda et. al (2005), Non-linear predictive control for a distillation column', 44th IEEE Conference on Decision and Control, Spain. pp. 5156- 5161. 
[9] J. D. Morn J. D. Morningred, B. E. Paden, D. E. Seborg, and D. A. Mellichamp, "An adaptive nonlinear predictive controller," Chem. Eng. Sci., vol. 47, pp. 755-762, 1992.

[10] Y. Nakamori, K. Suzuki, and T. Yamanaka, "Model predictive control using fuzzy dynamic models," in Proc. IFSA'91 Brussels, vol. 135, Brussels, Belgium, July 1991, p. 138. vol. Engrg..

R. Sivakumar is presently working as Associate Professor in the department of Electronics and Instrumentation Engineering, St. Joseph's College of Engineering, Anna University, Chennai. His areas of interest include Fuzzy Control, Model Predictive Control, Neural Networks, Computer based Process control etc

K. Suresh Manic is presently working as Assistant Professor in the department of Instrumentation and Control Engineering, St. Joseph's College of Engineering, Anna University, Chennai. His areas of interest include fault tolerant system design, Fuzzy controller, Evolvable hardware and Process control

V. Nerthiga is a research scholar in the department of Chemical Engineering at Anna University, Chennai. Her areas of interest include System Modelling, Energy modelling, Fuzzy logic, etc.

R. Akila is presently a research scholar department of Chemical Engineering at Anna University, Chennai. Her areas of interest are System Identification, Numerical methods and Mathematical modelling.

K. Balu is presently working as Professor in A.C. College of Technology, Chennai. He completed his doctorate from IIT Madras. His areas of interest include mathematical modeling, Fuzzy control, Evolvable hardware and process control etc 